\title{
Effect of triptorelin on lower urinary tract symptoms in Australian prostate cancer patients
}

\author{
Henry H Woo' \\ Declan G Murphy 2,3 \\ Gerard M Testa ${ }^{4}$ \\ Jeremy P Grummet ${ }^{5}$ \\ Michael Chong ${ }^{6}$ \\ Andrew P Stork ${ }^{7}$ \\ 'Sydney Adventist Hospital Clinical \\ School, University of Sydney, \\ Wahroonga, NSW, ${ }^{2}$ Division of Cancer \\ Surgery, Peter MacCallum Cancer \\ Centre, Melbourne, VIC, ${ }^{3}$ Australian \\ Prostate Cancer Research Centre, \\ Epworth Healthcare, Richmond, \\ VIC, ${ }^{4}$ Shire Urology, Miranda, NSW, \\ ${ }^{5}$ Australian Urology Associates, \\ Malvern, VIC, ${ }^{6}$ Department of Urology, \\ Repatriation General Hospital, \\ Daw Park, SA, ' Ipsen Pty Ltd, Glen \\ Waverley, VIC, Australia
}

This article was published in the following Dove Press journal:

Research and Reports in Urology

23 February 2017

Number of times this article has been viewed

Purpose: Prostate cancer is often comorbidly associated with lower urinary tract symptoms (LUTS), but few studies have assessed the effects of androgen deprivation therapy on LUTS in this patient group.

Patients and methods: We conducted a prospective, noninterventional, multicenter, observational study to assess the effectiveness of triptorelin ( $11.25 \mathrm{mg}$ every 12 weeks) over 48 weeks in men presenting with local stage T3/4 prostate cancer and moderate to severe LUTS (International Prostate Symptom Score [IPSS] >7) in a routine practice setting in Australia.

Results: Of the 44 men who enrolled, effectiveness data were available for 39 men. By the end of the study, $30 \%$ of men no longer met the IPSS criteria for moderate to severe LUTS. The proportion of patients with moderate to severe LUTS was $69.6 \%(16 / 23)$ at week 48 and $76.9 \%$ (30/39) at the last available visit (coprimary outcomes). An IPSS reduction of $\geq 3$ from week 0 was observed in $47 \%$ of men at week $4,56 \%$ at week $24,61 \%(14 / 23)$ at week 48 , and $61.5 \%$ $(24 / 39)$ at the last available visit. Quality of life was rated as mostly satisfied-to-delighted by $39.5 \%$ of patients at week $0,53.9 \%$ at week 24 , and $77.3 \%$ at week 48 . Triptorelin was well tolerated with 8 treatment-related adverse events reported, half of which were hot flushes; 5 patients discontinued due to the reported treatment-related adverse events.

Conclusion: This observational study suggests that triptorelin improves moderate to severe LUTS in prostate cancer patients in a routine clinical practice setting.

Keywords: GnRH agonist, IPSS

\section{Introduction}

The efficacy of androgen deprivation therapy (ADT) with luteinizing hormone-releasing hormone (LHRH) agonists has been widely reported in patients with advanced prostate cancer, ${ }^{1-4}$ but few studies have assessed the effects of ADT on lower urinary tract symptoms (LUTS) in this patient group.

LUTS most commonly arise not only from benign prostatic obstruction but also from obstruction due to locally advanced prostate cancer. Although it has been estimated that as many as $40 \%$ or more of men with prostate cancer will have moderate to severe LUTS, a number of cases of LUTS will be due to concomitant benign prostatic hypertrophy $(\mathrm{BPH})$ rather than prostate cancer. ${ }^{5-8}$

Triptorelin, an agonist analog of natural gonadotropin-releasing hormone (GnRH) with increased duration of action and increased affinity for the pituitary receptor as compared to the parent compound, is a widely used LHRH agonist with an established efficacy and safety profile in the treatment of prostate cancer. ${ }^{4-12}$ It causes
Correspondence: Henry H Woo San Clinic, Sydney Adventist Hospital, I85 Fox Valley Road, NSW 2076, Australia

Tel +6I 294738765

Fax +6I 294738969

Email henry.woo@sydney.edu.au 
downregulation of $\mathrm{GnRH}$ receptor number and postreceptor desensitization of the gonadotropic cell, resulting in reversible biochemical castration. Data on the impact of ADT (including triptorelin) on LUTS were, however, limited at the time of this study. The intention of this study was to evaluate the effectiveness of triptorelin on LUTS in patients with locally advanced or metastatic prostate cancer in routine clinical practice in Australia.

\section{Patients and methods}

\section{Ethics and regulatory}

This study was noninterventional and therefore falls outside the scope of the European Union (EU) Directive 2001/20/EC and the EU Directive 2005/28/EC. This study was conducted in compliance with the recommendations of the Declaration of Helsinki, 2008, and the International Ethical Guidelines for Epidemiological Studies (issued by the Council for International Organizations of Medical Sciences, February 2008), and adhered to all relevant Australian regulatory requirements. The study protocol and all other applicable documents were reviewed and approved by local independent ethics committees before start of patient recruitment at each site (Table S1). Written informed consent to participate in this study was obtained from all patients. The study was prospectively registered on the Australian New Zealand Clinical Trials Registry (ACTRN12610000558022).

\section{Study design}

This was a prospective, noninterventional, multicenter study to evaluate the effect of triptorelin on LUTS in prostate cancer patients scheduled to receive triptorelin embonate $11.25 \mathrm{mg}$. As this was an observational study, there was no randomization to treatment. Each patient was to receive 4 injections of triptorelin $11.25 \mathrm{mg}$, according to a schedule of 1 injection every 12 weeks (at baseline and 12, 24, and 36 weeks after baseline).

The patients were followed up for 48 weeks after start of treatment and attended 3 study visits: Visit 1 (baseline), Visit 2 (24 weeks after start of treatment), and Visit 3 (48 weeks after start of treatment). All procedures performed at these visits were in accordance with routine clinical practice.

The presence of LUTS was evaluated by patient completion of the International Prostate Symptom Score (IPSS) questionnaire $^{13}$ at baseline and at 1 month (completed by patients outside of study visits) and 24 and 48 weeks after start of triptorelin treatment. Additionally, total prostate-specific antigen (PSA) was measured at baseline and 24 and 48 weeks after baseline if part of the local standard clinical practice.

\section{Patients}

Patients were eligible for participation in the study if they had locally advanced or metastatic prostate cancer (at least local stage T3 disease), were scheduled to receive triptorelin $11.25 \mathrm{mg}$, and presented with moderate to severe LUTS (IPSS score $>7$ ). Patients could receive concomitant antiandrogen treatment to prevent flare up, at treatment initiation, according to current clinical practice.

Exclusion criteria included therapy with an LHRH analog in the last 6 months, life expectancy $<12$ months, risk of a serious complication in the case of tumor flare (vertebral metastases threatening spinal cord compression or significant obstructive uropathy), treatment with a 5-alpha reductase inhibitor in the last 6 months, treatment with alpha receptor blockers in the 2 weeks prior to the start of the study, surgery for the treatment of LUTS, history of surgical castration, and history of urethral stricture.

\section{Treatment}

Triptorelin $11.25 \mathrm{mg}$ (as embonate salt; Diphereline; Ipsen Pty Ltd, Glen Waverley, VIC, Australia) was administered as described in the product information approved in Australia.

Each eligible patient was to receive 4 intramuscular injections of triptorelin $11.25 \mathrm{mg}$, according to a schedule of 1 injection administered every 12 weeks (at baseline, week 12, week 24, and week 36).

\section{Assessments}

The primary end point was the number and percentage of patients having moderate to severe LUTS (IPSS total score $>7$ ) at week 48 and at last available visit. The IPSS questionnaire consists of 7 symptom questions (1 each on incomplete emptying, frequency, intermittency, urgency, weak stream, straining, and nocturia) and 1 quality of life question ("If you were to spend the rest of your life with your urinary condition the way it is now, how would you feel about that?"). The 7 symptom questions are self-rated from 0 (not at all) to 5 (almost always). A total symptom score of 0 is considered asymptomatic, $1-7$ mildly symptomatic, 8-19 moderately symptomatic, and 20-35 as severely symptomatic. A 3-point improvement in total score is considered clinically meaningful. ${ }^{14}$

Secondary end points included the number and percentage of patients having moderate to severe LUTS at week 4 and week 24; IPSS total score, IPSS storage (previously irritative) subscore, IPSS voiding (previously obstructive) subscore at baseline and at each time point; PSA result $(\mathrm{ng} / \mathrm{mL})$ and PSA categories at baseline and at each time 
point; correlation between changes in IPSS and PSA result $(\mathrm{ng} / \mathrm{mL})$ and correlation between changes in IPSS score categories and PSA result $(\mathrm{ng} / \mathrm{mL})$; quality of life question of IPSS at baseline and at each time point. Any related adverse events during the study were reported directly to the drug manufacturer. Safety data were not collected in the case report forms.

\section{Statistics}

The primary effectiveness analysis was based on the effectiveness population as well as the per protocol population. The secondary effectiveness analyses were performed on the effectiveness population. All statistical tests were exploratory and 2 -sided at the $5 \%$ level of significance. In addition, 95\% confidence intervals (CIs) of the proportions described in the end points have been calculated. Approximate binomial CIs have been produced using the Agresti-Coull method. A paired $t$-test was used to test if the mean change from baseline in PSA at each post-baseline time point differed from 0 . Bhapkar's test was used for the analyses of distribution shifts between baseline and post-baseline time points of IPSS categories and PSA categories. Pearson's correlation coefficient was used for the correlation analyses.

The primary end point, number and percentage of patients having moderate to severe LUTS (defined as IPSS total score $>7$ ) at week 48 and at last available visit, was presented using descriptive statistics including 95\% CIs for the percentage.

\section{Results \\ Patient enrollment and baseline characteristics}

The study initially planned to enroll 70 patients; however, after trying for 2.5 years to identify eligible patients, the investigators mutually agreed to close recruitment. In total, 44 patients were enrolled into the study at 9 sites in Australia. Between 1 and 8 patients were enrolled at each site. The first patient was enrolled on March 8, 2011, and the last patient completed the study on May 1, 2014. Forty-three of the 44 patients enrolled (97.7\%) received at least 1 injection of triptorelin during the study and 23 patients (52.3\%) completed all study visits. Overall, 39 men were included in the effectiveness population. The effectiveness population was defined as all patients who received at least 1 injection and had a post-baseline IPSS score (Figure 1). Baseline characteristics of the effectiveness population are summarized in Table 1. The mean ( \pm standard deviation [SD]) age was $75.6 \pm 8$ years, the mean weight was $79.8 \pm 13.9 \mathrm{~kg}$, and the mean BMI was $26.7 \pm 3.9 \mathrm{~kg} / \mathrm{m}^{2}$. All patients had moderate to severe LUTS at baseline, and 5 received a short course (1 month) of an antiandrogen agent (bicalutamide) at triptorelin initiation, to prevent potential testosterone flare effects.

\section{Primary analysis}

Triptorelin reduced the proportion of patients with moderate to severe LUTS (IPSS >7) during the study (Figure 2). In the effectiveness population, the proportion of patients with moderate to severe LUTS at week 48 was $69.6 \%$ (16/23; $95 \%$ CI: $48.9 \%-84.6 \%)$. Seven patients (30.4\%) had shifted from

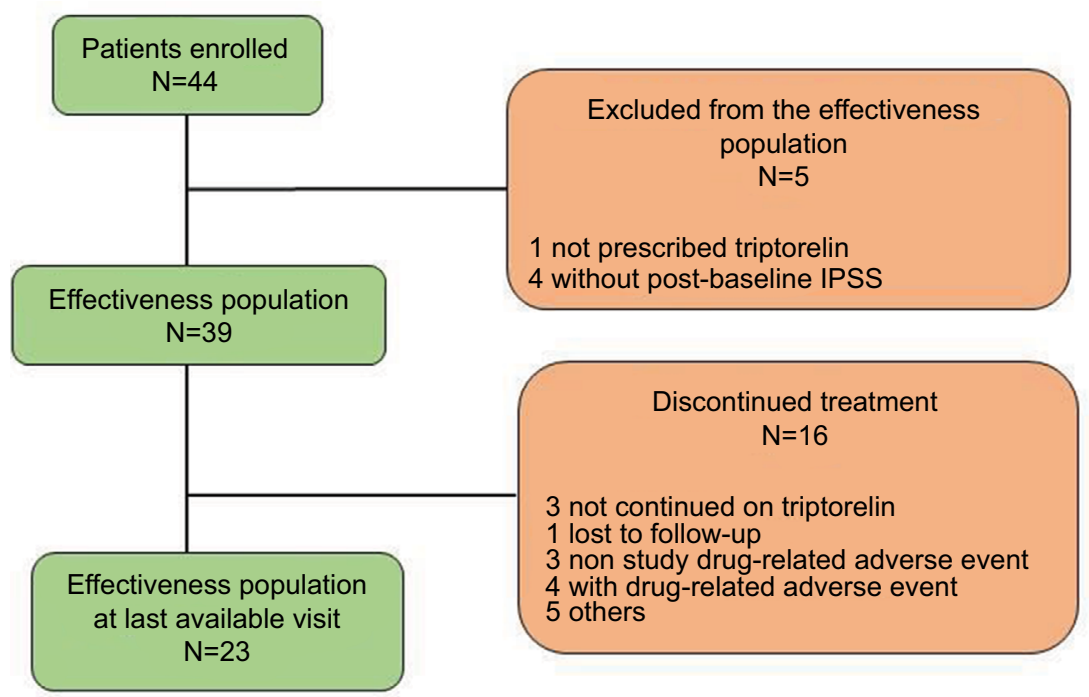

Figure I Patient disposition.

Abbreviation: IPSS, International Prostate Symptom Score. 
Table I Baseline characteristics of effectiveness population

\begin{tabular}{|c|c|}
\hline Variable & $\begin{array}{l}\text { Patients }(\mathrm{N}=39 \text { unless } \\
\text { otherwise stated) }\end{array}$ \\
\hline Age in years, mean (SD) & $75.6(8.0)$ \\
\hline BMI $\left(\mathrm{kg} / \mathrm{m}^{2}\right)$, mean $(\mathrm{SD})^{\mathrm{a}}$ & $26.7(3.9)$ \\
\hline \multicolumn{2}{|l|}{ Prostate cancer stage, n (\%) } \\
\hline T3 & $30(76.9)$ \\
\hline $\mathrm{T} 4$ & $9(23.1)$ \\
\hline \multicolumn{2}{|l|}{ Gleason score, n (\%) ${ }^{\mathrm{b}}$} \\
\hline 6 & $2(6.8)$ \\
\hline 7 & $13(44.8)$ \\
\hline 8 & $2(6.8)$ \\
\hline 9 & II (37.9) \\
\hline 10 & I (3.5) \\
\hline Patients with metastasis (MI), n (\%) & $15(38.5)$ \\
\hline \multicolumn{2}{|l|}{ PSA, ng/mL, n (\%) } \\
\hline 0 to $<4$ & I (2.9) \\
\hline$\geq 4$ to 10 & $9(25.7)$ \\
\hline$\geq 10$ & $25(71.4)$ \\
\hline $\begin{array}{l}\text { Time since diagnosis, years, median } \\
(\min , \max )^{d}\end{array}$ & $0.12(0.0,9.4)$ \\
\hline $\begin{array}{l}\text { Hormonal therapy history for prostate } \\
\text { cancer, } \mathrm{n}(\%)\end{array}$ & $6(15.4)$ \\
\hline $\begin{array}{l}\text { Radiation therapy history for prostate } \\
\text { cancer, } \mathrm{n}(\%)\end{array}$ & $3(7.7)$ \\
\hline \multicolumn{2}{|l|}{ IPSS category, n (\%) } \\
\hline Moderate & $31(79.5)$ \\
\hline Severe & $8(20.5)$ \\
\hline
\end{tabular}

Notes: ${ }^{\mathrm{a}} \mathrm{N}=3 \mathrm{I}$; ${ }^{\mathrm{b}} \mathrm{N}=29 ;{ }^{\mathrm{c}} \mathrm{N}=35$ and ${ }^{\mathrm{d}} \mathrm{N}=36$. $\mathrm{N}$, total number.

Abbreviations: BMI, body mass index; SD, standard deviation; PSA, prostatespecific antigen; IPSS, International Prostate Symptom Score; n, number.

moderate/severe to mild LUTS, as compared to baseline. At the last available visit, $76.9 \%$ of the patients $(30 / 39$; 95\% CI: $61.5 \%-87.6 \%$ ) had moderate to severe LUTS.
Nine patients $(23.1 \%)$ had shifted from moderate/severe to mild LUTS, as compared to baseline. Similar results were observed in the per protocol population as compared to the effectiveness population.

Based on descriptive analysis, the IPSS total score \pm SD reduced from an average of $16.1 \pm 6.5$ at baseline to $10.5 \pm 5.8$ at week $48(n=23)$ and $11.9 \pm 6.7$ at the last available visit $(n=39)$, ie, the mean change from baseline \pm SD over the course of the study was $-4.2 \pm 7.0$ (median -4.0 ), indicating an improvement.

An IPSS reduction of $\geq 3$ from baseline was observed in $61 \%(14 / 23)$ of patients for whom a score was available at week 48 and $61.5 \%(24 / 39)$ at the last available visit.

\section{Secondary analysis}

As shown in Figure 2, the proportion of patients with moderate to severe LUTS in the effectiveness population at month 1 was $66.7 \%(10 / 15 ; 95 \%$ CI: 41.5\%-85.0\%), although data were available for less than half of the patients at this time point. Four patients $(26.7 \%)$ had shifted from moderate to mild symptoms, 1 patient (6.7\%) from severe to mild symptoms, and 1 patient $(6.7 \%)$ from severe to moderate. The proportion of patients with moderate to severe LUTS at week 24 was 74.4\% (29/39; 95\% CI: 58.8\%-85.6\%). Nine patients $(23.1 \%)$ had shifted from moderate to mild symptoms, 1 patient $(2.6 \%)$ from moderate to no symptoms, and 4 patients from severe to moderate.

The mean IPSS total score \pm SD was $11.3 \pm 5.3$ at week 4 $(n=15)$ and 12.4 $\pm 7.3(n=39)$ at week 24. An IPSS reduction

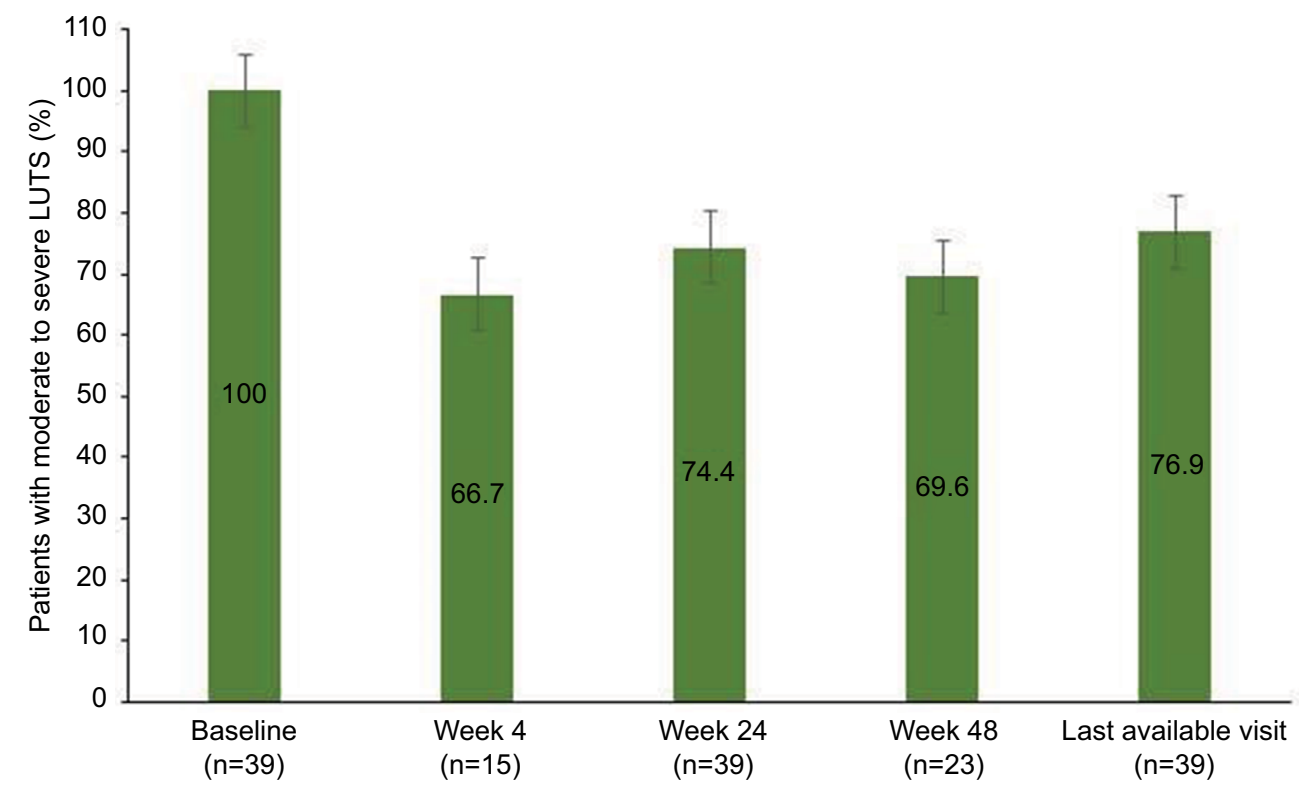

Figure 2 Proportion of patients with moderate to severe LUTS (according to total IPSS) at all time points in the effectiveness population. Abbreviations: LUTS, lower urinary tract symptoms; IPSS, International Prostate Symptom Score. 


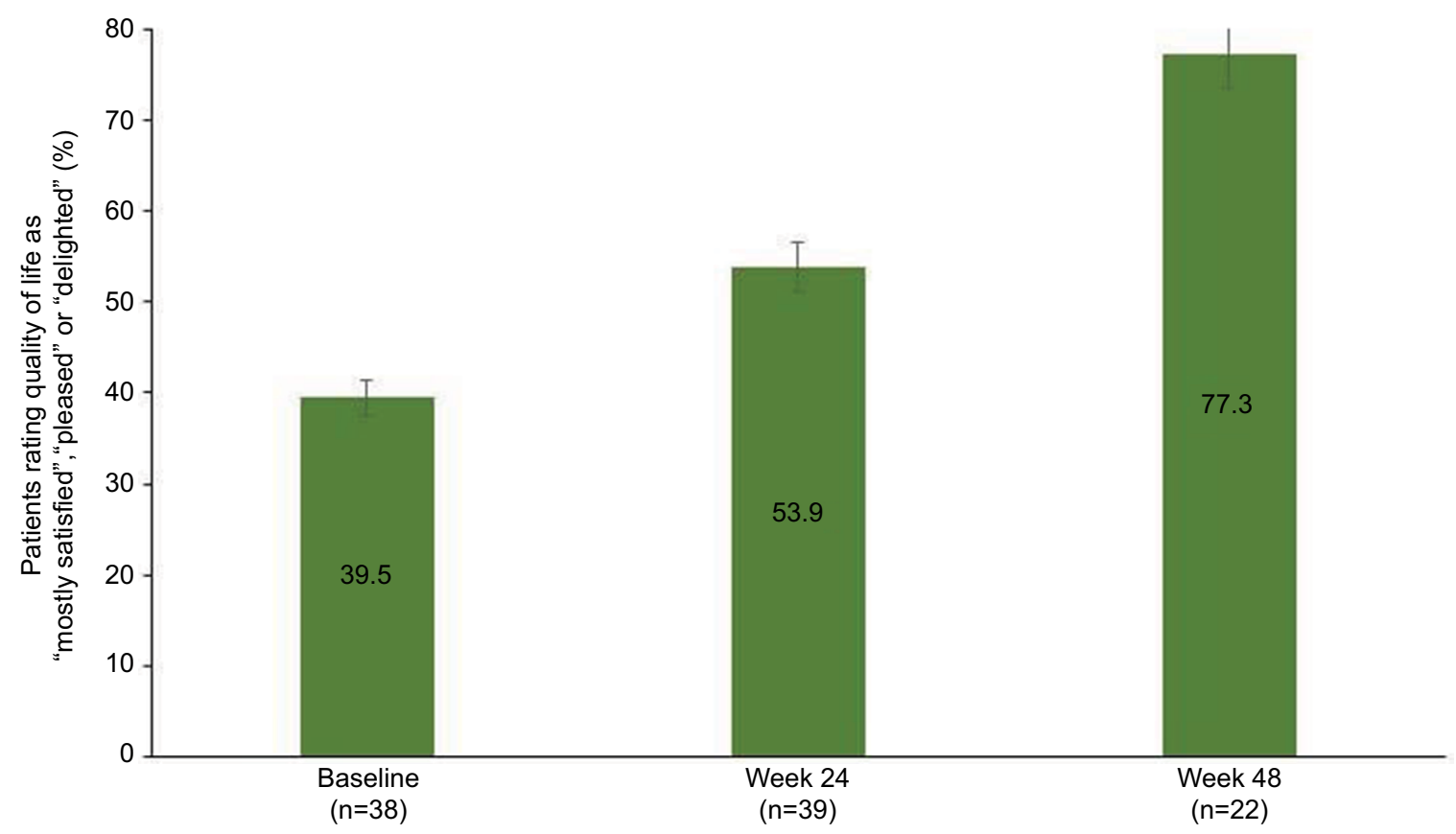

Figure 3 Changes in quality of life during the study.

of $\geq 3$ from baseline was observed in $47 \%$ of men at week 4 $(7 / 15)$ and $56 \%$ at week $24(22 / 39)$.

The mean IPSS voiding and storage subscores decreased over time, indicating an improvement. The mean IPSS voiding score decreased from 8.2 at baseline to 4.6 at week 48 $(n=23)$ and 5.6 at the last available visit $(n=39)$, ie, the mean change from baseline \pm SD over the course of the study was $-2.6 \pm 4.6$ (median -3.0 ). The mean storage score decreased from 7.9 at baseline to 5.9 at week $48(\mathrm{n}=23)$ and 6.3 at the last available visit $(\mathrm{n}=39)$, ie, the mean change from baseline \pm SD over the course of the study was $-1.7 \pm 3.5$ (median-2.0).

Based on descriptive analysis of the IPSS quality of life question, the proportion of patients using any of the first 3 answers ( 0 - Delighted, 1 - Pleased, and 2 - Mostly satisfied) at baseline, month 1 , week 24 , week 48 , and at last available visit were $39.5 \%(\mathrm{n}=38), 60.0 \%(\mathrm{n}=15), 53.9 \%(\mathrm{n}=39)$, $77.3 \%(n=22)$, and $66.7 \%(n=39)$, respectively, indicating an improvement from month 1 sustained during the full study course (Figure 3).

A statistically significant reduction (paired $t$-test) in mean PSA level in blood from baseline to week 24 and to last available visit was detected. The mean change \pm SD was $-47.9 \pm 113.3 \mathrm{ng} / \mathrm{mL}$ (median -14.7 ) from baseline to week $24(\mathrm{n}=33, p=0.0211),-51.6 \pm 129.8 \mathrm{ng} / \mathrm{mL}$ (median $-12.4)$ from baseline to week $48(\mathrm{n}=21, p=0.0838)$, and $-46.4 \pm 106.7 \mathrm{ng} / \mathrm{mL}$ (median -14.7 ) from baseline to last available visit $(\mathrm{n}=33, p=0.0179)$. The difference from baseline in PSA, based on shift between categories ( 0 to $<4$, $\geq 4$ to $<10, \geq 10 \mathrm{ng} / \mathrm{mL}$ ), was statistically significant at all post-baseline time points $(p<0.0001$, Bhapkar's test). Based on the fixed categories, a shift toward lower levels of PSA was seen over time.

Median (1st quartile, 3rd quartile) PSA levels $(\mathrm{ng} / \mathrm{mL})$ reduced from $18.0(9.8,56.0)$ at baseline to $1.0(0.3,3.9)$ at week 24 and $0.6(0.2,3.7)$ at week 48 (Figure 4$)$. No meaningful correlation between changes in IPSS score and PSA was demonstrated during the study $(p=0.071)$, although a weak correlation between change from baseline in total IPSS and change from baseline in PSA result $(\mathrm{ng} / \mathrm{mL})$ was found at week 48 (Pearson correlation coefficient $=0.10 ; p=0.6801$ ).

\section{Discussion}

This Australian study, despite being relatively small in patient numbers (a much lower than anticipated number of patients initiating or recommencing ADT also presented with sufficiently bothersome LUTS, ie, IPSS $>7$ ), suggests that triptorelin improves moderate to severe LUTS in patients with prostate cancer treated in routine clinical practice. The number of patients with moderate or severe LUTS was reduced at all time points in the study, and this reduction was maintained for up to 48 weeks of treatment. Over $60 \%$ of men reported a clinically meaningful improvement in their 


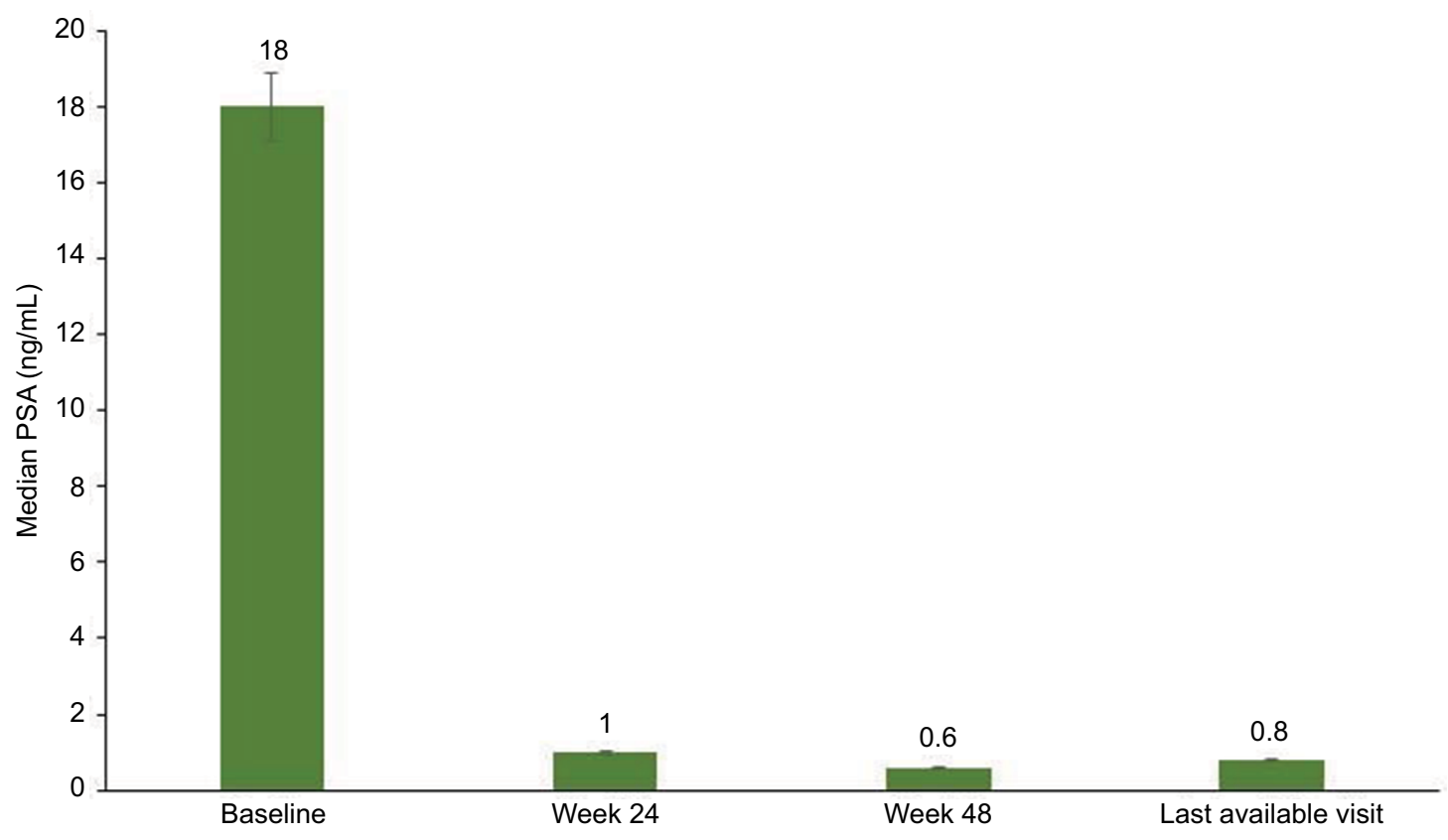

Figure 4 Median PSA at each time point in the effectiveness population. Abbreviation: PSA, prostate-specific antigen.

total symptom score. Improvements were also seen in the IPSS voiding and storage subscores and in quality of life. As expected, PSA levels were also reduced at 24 weeks of treatment with triptorelin and were maintained till the end of the study. Data on $Q_{\max }$ and residual volume, however, were available for a very limited number of patients, and no consistent conclusions on these parameters could be drawn based on the available data. No correlation between IPSS change and PSA change was observed during the study, most likely due to the low number of patients included.

At the time that this study was planned, data on the impact of ADT (including triptorelin) on LUTS were limited. Since the completion of this study, however, the results from a prospective grouped analysis of noninterventional, multicenter studies of LUTS have been published. ${ }^{15}$ These studies were located in Algeria, Belgium, China, Hungary, Romania, and South Korea, in patients who were scheduled to receive triptorelin (3-month or 1-month formulation) in clinical practice. The proportion of patients with moderate or severe LUTS $(n=1282)$, as assessed by IPSS, was reduced from baseline after 24, 48 weeks, and at last available visit. The reduction in moderate or severe LUTS with triptorelin treatment was significantly reduced (overall time effect, $p<0.0001$ ). The mean total IPSS decreased from 18.2 at baseline to 10.6 at week $48(p<0.001)$, the magnitude of which is clinically meaningful. Additionally, quality of life was improved with triptorelin treatment, as measured by a significant reduction in the adjusted mean quality of life score from 4.1 to 2.9 $(p<0.001)$ after 24 weeks and $2.5(p<0.001)$ after 48 weeks.
Incidentally, the recent publication from the Belgian cohort ${ }^{13}$ demonstrated results consistent with the main publication. Short-term (12 weeks) data on the LUTS impact of some other ADT agents also became available during the time that the abovementioned studies were being conducted. ${ }^{16}$

In spite of the lower numbers in our Australian study (and being lower than originally intended), and some differences in the patient populations, there is a steady consistency of results between this study, the grouped analysis, and the 325-patient Belgian study. All 3 studies report clinically meaningful reductions in IPSS in a proportion of prostate cancer patients with moderate to severe LUTS treated with triptorelin, with accompanying changes in severity group status proportions.

Being observational studies, the grouped analysis, Belgian cohort, and our Australian study have inherent limitations such as potential selection bias and lack of control for confounding factors. Although it has been used widely to assess LUTS, the IPSS relies on patient recall to rate symptoms. Our Australian study is also limited by the assumption that men with locally advanced prostate cancer with LUTS have their symptoms on the basis of obstruction due to cancer. It is quite possible that some nonresponders to ADT had LUTS on the basis of other etiology, eg, an overactive bladder. The advantage of these observation studies, however, is their real-world clinical setting, which provides an indication of the impact of triptorelin on LUTS in a heterogeneous population.

In the context of routine clinical practice, it is worth noting that BPH presents itself clinically as LUTS. The 
prevalence of prostate cancer and BPH increases with age, and there are similarities between the diseases, both being androgen dependent and responsive to ADT. Indeed, both diseases frequently coexist which brings to question whether LUTS is due to the cancer itself or BPH.

Both surgical and medical castrations have been used to treat urinary symptoms in men with BPH. Most studies have involved men with large prostates and, by virtue of the mechanism of action being reduction of prostate volume, these men would most likely respond to treatment. These studies have established that at least 6 months of treatment is necessary to achieve maximal reduction in prostate volume. ${ }^{17}$ While there has been no head-to-head comparison and, for that matter, very limited data in the prostate cancer population, differences in the magnitude of prostate volume reduction between $\mathrm{BPH}$ and prostate cancer have not been established. Clinical experience with treating men who have locally advanced prostate cancer with ADT recognizes that the prostate can reduce substantially in size. ${ }^{18}$ On the assumption that the urinary symptoms associated with prostate cancer are due to mechanical obstruction, it is reasonable to suggest that LUTS in the setting of locally advanced prostate cancer should respond to ADT.

\section{Conclusion}

Despite its limitations, this Australian study corroborates well with the multicountry-grouped analysis, and together the results indicate that triptorelin improves moderate to severe LUTS in patients with prostate cancer treated in routine clinical practice, with the number of patients with moderate or severe LUTS reduced at all time points for up to 48 weeks of treatment.

\section{Acknowledgments}

The authors acknowledge and thank the other participating sites and coinvestigators: Anthony Costello (Epworth Hospital, Richmond, VIC, Australia), Tru Ngo (Shire Urology, Miranda, NSW, Australia), Darren Foreman and Kym Horsell (Repatriation General Hospital, Daw Park, SA, Australia), Kayvan Haghighi and Pascal Mancuso (Macarthur Urology, Campbelltown, NSW, Australia), John Yaxley (Brisbane Private Hospital, Brisbane, QLD, Australia ), Steven Sowter (Riverina Urology, Wagga Wagga, NSW, Australia), and Andrew Tan (Mount Urology, Perth, WA, Australia). Additionally, the authors acknowledge and thank the patients who agreed to participate in this study. Data management and statistical analysis support was provided by PCG Clinical Services (Uppsala, Sweden), including use of Viedoc for data collection. The authors acknowledge and thank Kim
Magner of Lateral Connections (Melbourne, VIC, Australia) for medical writing support in developing the manuscript. The study was funded by Ipsen Pty Ltd.

\section{Author contributions}

HHW, DGM, GMT, JPG, and MC participated in study design and data collection. All authors contributed toward data analysis, drafting and critically revising the paper and agree to be accountable for all aspects of the work.

\section{Disclosure}

DGM has received honoraria for speaking and consulting services from Ipsen Pty Ltd. JPG has received a research grant and honoraria for consulting services from Ipsen Pty Ltd. APS is an employee of Ipsen Pty Ltd. The authors report no other conflicts of interest in this work.

\section{References}

1. Labrie F, Belanger A, Luu-The V, et al. Gonadotropin-releasing hormone agonists in the treatment of prostate cancer. Endocr Rev. 2005;26(3):361-379.

2. Harris WP, Mostaghel EA, Nelson PS, Montgomery B. Androgen deprivation therapy: progress in understanding mechanisms of resistance and optimizing androgen depletion. Nat Clin Pract Urol. 2009;6(2): 76-85.

3. Schroeder FH, Hugosson J, Roobol MJ, et al; ERSPC Investigators. Prostate-cancer mortality at 11 years of follow-up. $N$ Engl $\mathrm{J} \mathrm{Med}$. 2012;366(11):981-990.

4. Lundstrom EA, Rencken RK, van Wyk JH, et al. Triptorelin 6-month formulation in the management of patients with locally advanced and metastatic prostate cancer: an open-label, non-comparative, multicentre, phase III study. Clin Drug Investig. 2009;29(12):757-765.

5. Andersson SO, Rashidkhani B, Karlberg L, Wolk A, Johansson JE. Prevalence of lower urinary tract symptoms in men aged 45-79 years: a population-based study of 40000 Swedish men. BJU Int. 2004;94(3):327-331.

6. Guess HA. Benign prostatic hyperplasia and prostate cancer. Epidemiol Rev. 2001;23:152-158.

7. Hamilton W, Sharp D. Symptomatic diagnosis of prostate cancer in primary care: a structured review. Br J Gen Pract. 2004;54(505): 617-621.

8. Lehrer S, Stone NN, Droller MJ, Stock RG. Association between American Urologic Association (AUA) urinary symptom score and disease stage in men with localized prostate cancer. Urol Oncol. 2002;7(2): 73-76.

9. Heyns CF, Simonin MP, Grosgurin P, Schall R, Porchet HC; South African Triptorelin Study Group. Comparative efficacy of triptorelin pamoate and leuprolide acetate in men with advanced prostate cancer. BJU Int. 2003;92(3):226-231.

10. Teillac P, Heyns CF, Kaisary AV, Bouchot O, Blumberg J. Pharmacodynamic equivalence of a decapeptyl 3-month SR formulation with the 28-day SR formulation in patients with advanced prostate cancer. Horm Res. 2004;62(5):252-258.

11. Martinez-Pineiro L, Schalken JA, Cabri P, Maisonobe P, de la Taille A; Triptocare Study Group. Evaluation of urinary prostate cancer antigen-3 (PCA3) and TMPRSS2-ERG score changes when starting androgendeprivation therapy with triptorelin 6-month formulation in patients with locally advanced and metastatic prostate cancer. BJU Int. 2013; 114(4):608-616.

12. Ploussard G, Mongiat-Artus P. Triptorelin in the management of prostate cancer. Future Oncol. 2013;9(1):93-102. 
13. Peltier A, Aoun F, De Ruyter V, Cabri P, Van Velthoven R. Triptorelin in the relief of lower urinary tract symptoms in advanced prostate cancer patient: the RESULT study. Prostate Cancer. 2015;2015:978194.

14. Bosch JL, Hop WC, Kirkels WJ, Schroder FH. The International Prostate Symptom Score in a community-based sample of men between 55 and 74 years of age: prevalence and correlation of symptoms with age, prostate volume, flow rate and residual urine volume. Br J Urol. 1995;75(5):622-630.

15. Gil T, Aoun F, Cabri P, Maisonobe P, van Velthoven R. A prospective, observational grouped analysis to evaluate the effect of triptorelin on lower urinary tract symptoms in patients with advanced prostate cancer. Ther Adv Urol. 2015;7(3):116-124.
16. Cui Y, Zong H, Yan H, Li N, Zhang Y. Degarelix versus goserelin plus bicalutamide therapy for lower urinary tract symptom relief, prostate volume reduction and quality of life improvement in men with prostate cancer: a systemic review and meta-analysis. Urol Int. 2014; 93:152-159.

17. Peters CA, Walsh PC. The effect of nafarelin acetate, a luteinizinghormone-releasing hormone agonist, on benign prostatic hyperplasia. N Engl J Med. 1987;317(10):599-604.

18. Padhani AR, MacVicar AD, Gapinsky CJ, et al. Effects of androgen deprivation on prostatic morphology and vascular permeability evaluated with MR imaging. Radiology. 2001;218(2):365-374. 


\section{Supplementary material}

Table SI Participating sites, investigators, and approving ethics committees

\begin{tabular}{lll}
\hline Approving local human research ethics committee (HREC) & Site name & Site lead investigator \\
\hline Bellberry Limited HREC & Australian Urology Associates & A/Prof J Grummet \\
& Brisbane Private Hospital & Dr Yaxley \\
& Macarthur Urology & Dr G Testa \\
Epworth HealthCare HREC & Shire Urology & A/Prof D Murphy \\
Mount Hospital HREC & Epworth Richmond & Dr A Tan \\
Riverina Cancer Care Centre HREC & Mount Urology & Dr S Sowter \\
Southern Adelaide Health Service/Flinders University HREC & Riverina Cancer Care Centre & Dr M Chong \\
Sydney Adventist Hospital HREC & Repatriation General Hospital & A/Prof H Woo \\
\hline
\end{tabular}

Research and Reports in Urology is an international, peer-reviewed, open access journal publishing original research, reports, editorials, reviews and commentaries on all aspects of adult and pediatric urology in the clinic and laboratory including the following topics: Pathology, pathophysiology of urological disease; Investigation and treatment of urological disease; Pharmacology of drugs used for the treatment of urological disease. The manuscript management system is completely online and includes a very quick and fair peer-review system, which is all easy to use. Visit http://www.dovepress.com/testimonials.php to read real quotes from published authors. 